\title{
VARIATION CONVERGENCE FOR BERNSTEIN POLYNOMIALS
}

\author{
MARTIN PRICE ${ }^{1}$
}

1. Introduction. In this paper we investigate the convergence of the variations of Bernstein polynomials. The $n$th Bernstein polynomial of the real function $f$ is defined to be

$$
B_{n} f(x) \equiv \sum_{r=0}^{n} f(r / n) p_{n r}(x) \quad \text { where } \quad p_{n r}(x) \equiv C_{n, r} x^{r}(1-x)^{n-r}
$$

for $x \in[0,1]$. We denote the total variation of $B_{n} f$ by $\mathrm{V} B_{n} f$. It is shown in [3] that for any $f$ and $n$,

$$
\mathrm{V} B_{n} f \leqq \sum_{r=0}^{n-1}\left|f\left(\frac{r+1}{n}\right)-f\left(\frac{r}{n}\right)\right| \leqq \mathrm{V} f,
$$

and also that

TheOREM (1.1). If $f$ is of bounded variation, then $\mathrm{V} B_{n} f$ converges to the variation of $f$, computed only over points of continuity of $f$.

In view of the fact that $B_{n} f$ depends only on the rational values of $f$, we have considered functions $f$, defined only on the rationals, which we call "skeletons," as in the paper of Herzog and Hill [2]. For functions $f$ and $g$, we have

$$
\left|\mathrm{V} B_{n} f-\mathrm{V} B_{n} g\right| \leqq \mathrm{V}\left(B_{n} f-B_{n} g\right)=\mathrm{V} B_{n}(f-g) .
$$

Hence we study conditions under which $\mathrm{V} B_{n} f \rightarrow 0$ for a single function. Herzog and Hill showed that if $f$ satisfies

$$
\sum_{r=0}^{n}\left|f\left(\frac{r}{n}\right)\right|=o\left(n^{1 / 2}\right)
$$

then $B_{n} f \rightarrow 0$ for each $x$ in $(0,1)$. Our result is that if $f$ vanishes in a neighborhood of 0 and 1 , and if (1.4) holds, then $\mathrm{V} B_{n} f \rightarrow 0$. Furthermore, if the sums are $O\left(n^{1 / 2}\right)$, then the $\mathrm{V} B_{n} f$ are bounded. Finally, there exist bounded skeletons such that the above sums are $O\left(n^{1 / 2} A_{n}\right)$ for an arbitrary monotone sequence $A_{n} \rightarrow+\infty$ but such that $\mathrm{V} B_{n_{k}} f$ $\rightarrow+\infty$ for a subsequence $\left\{n_{k}\right\}$.

Received by the editors September 14, 1966 and, in revised form, March 20, 1967.

1 This is a part of the author's Ph.D. thesis at Purdue University. The work was given support by N.S.F. Grant \#GPO3515. The author is indebted to the referee for suggesting an improvement in the second part of Theorem (3.1). 
2. Preliminary notions. Most of the facts which will be needed can be found in [2] or [3]. In particular, we need the following:

REMARK (2.1). For all $n, \sum_{r=0}^{n} p_{n r}(x)=1, B_{n} f(0)=f(0)$ and $B_{n} f(1)$ $=f(1)$. (Thus a necessary condition for $\mathrm{V} B_{n} f \rightarrow 0$ is $f(0)=f(1)$.)

REMARK (2.2). If $\delta>0, x \in[0,1]$ then

$$
\sum_{|r / n-x| \geq \delta} p_{n r}(x) \leqq \frac{1}{4 n \delta^{2}} .
$$

REMARK (2.3). For any $x \in(0,1)$ and $r=0,1, \cdots, n$

$$
p_{n r}(x) \leqq C /[n x(1-x)]^{1 / 2}
$$

where $C$ is an absolute constant.

REMARK (2.4). For all $n$ and $f$

$$
\mathrm{V} B_{n} f \leqq \sum_{r=0}^{n}\left|f\left(\frac{r}{n}\right)\right| \mathrm{V} p_{n r}(x) \leqq 2 \sum_{r=0}^{n}\left|f\left(\frac{r}{n}\right)\right| \operatorname{Max} p_{n r}
$$

where the Max is taken over $x \in[0,1]$. The second inequality holds since $p_{n 0}$ and $p_{n n}$ are monotone, and $p_{n r}, r=1, \cdots, n-1$ have a single peak at $x=r / n$.

\section{A theorem on the convergence of $\mathrm{V} B_{n} f$.}

Theorem (3.1). Suppose $f$ vanishes off the interval $I_{A}=[A, 1-A]$ for some $0<A \leqq \frac{1}{2}$. Then if (a)

$$
\sum_{r=0}^{n}\left|f\left(\frac{r}{n}\right)\right|=o\left(n^{1 / 2}\right)
$$

$\mathrm{V} B_{n} f \rightarrow 0$; if (b) the above sums are $O\left(n^{1 / 2}\right)$, then $\mathrm{V} B_{n} f<M$ for all $n$. Furthermore, (c) there is a bounded skeleton vanishing off some $I_{A}$ with the sums $O\left(n^{1 / 2}\right)$, and such that $\mathrm{V} B_{n_{k}} f>M$ for some $M>0$ and a subsequence $\left\{n_{k}\right\}$. Finally, (d) there is a bounded skeleton $f$ which vanishes off some $I_{A}$ with the above sums of order $O\left(n^{1 / 2} A_{n}\right)$ for monotone sequences $A_{n} \rightarrow+\infty$ however slowly, but such that $\mathrm{V} B_{n} f$ is unbounded.

Proof. By Remark (2.4)

$$
\mathrm{V} B_{n} f \leqq 2 \sum_{r=0}^{n}\left|f\left(\frac{r}{n}\right)\right| \operatorname{Max} p_{n r}=2 \sum_{r / n \in I \boldsymbol{\Delta}}\left|f\left(\frac{r}{n}\right)\right| \operatorname{Max} p_{n r} .
$$

If we let $x=r / n$, it follows from Remark (2.3) that

$$
\operatorname{Max} p_{n r}<C n^{1 / 2} /[r(n-r)]^{1 / 2}
$$

for $r=1, \cdots, n-1$, since for these $r, p_{n r}(0)=p_{n r}(1)=0$. If $r / n \in I_{A}$, 
then $r>n A$ and $n-r \geqq n A$. Hence for $r / n \in I_{\boldsymbol{A}}$,

$$
\operatorname{Max} p_{n r} \leqq C n^{1 / 2} /[n A \cdot n A]^{1 / 2}=C / A n^{1 / 2} .
$$

Substitution in (3.2) gives

$$
\mathrm{V} B_{n} f \leqq \frac{2 C}{A n^{1 / 2}} \sum_{r / n \in I_{\boldsymbol{A}}}\left|f\left(\frac{r}{n}\right)\right| .
$$

Parts (a) and (b) of the theorem now follow directly from (3.5).

We now prove (d). We can assume $A_{n} n^{-1 / 2} \leqq 1$. Let $I_{A} \equiv[1 / 4,3 / 4]$. If $\delta_{n} \equiv n^{-1 / 2}$, then for each $n, I_{A}$ contains $m=\left[n^{1 / 2} / 4\right]$ adjacent nonoverlapping subintervals $I_{k}, k=1, \cdots, m$ of length $2 \delta_{n}$. First we define a "partial" skeleton $g_{n}$. If $r / n \in I_{k}$ with $k$ even, we put $g_{n}(r / n)=1$ and for all other rationals, put $g_{n}=0$. Then if $x$ is the midpoint of an $I_{k}$, with $k$ even,

$$
\begin{aligned}
B_{n} g_{n}(x) & \geqq \sum_{|r / n-x| \leq \delta_{n}} p_{n r}(x)=1-\sum_{|r / n-x|>\delta_{n}} p_{n r}(x) \\
& \geqq 1-\frac{1}{4 n \delta_{n}^{2}}=\frac{3}{4}
\end{aligned}
$$

because of Remarks (2.1) and (2.2). Also, if $x^{\prime}$ is the midpoint of an $I_{k}$, for $k_{\text {odd, then }}^{7}$

$$
B_{n} g_{n}\left(x^{\prime}\right) \leqq \sum_{\left|r / n-x^{\prime}\right| \geq \delta_{n}} p_{n r}\left(x^{\prime}\right) \leqq \frac{1}{4} .
$$

Hence the oscillation of $B_{n} g_{n}(x)$ on $I_{k} \cup I_{k+1}$ is at least $\frac{1}{2}$, so that

$$
\mathrm{V} B_{n} g_{n} \geqq \frac{1}{2}(m-1) \geqq \frac{1}{10} n^{1 / 2}
$$

for large $n$; also $\sum_{r=0}^{n} g_{n}(r / n)<n$. Next we modify the $g_{n}$ by putting $f_{n}=A_{n} n^{-1 / 2} g_{n}$. Then $0 \leqq f_{n} \leqq 1$ on $[0,1]$ and

$$
\mathrm{V} B_{n} f_{n} \geqq \frac{1}{10} A_{n}, \quad \sum_{r=0}^{n} f_{n}\left(\frac{r}{n}\right) \leqq A_{n} n^{1 / 2} .
$$

Now choose $\left\{n_{k}\right\}$ to be a sequence of relatively prime integers increasing so rapidly that

$$
n_{k-1}^{1 / 2} A_{n_{k-1}} \leqq \frac{1}{2} n_{k}^{1 / 2} A_{n_{k}} \text {. }
$$

Define a skeleton $f$ by putting $f\left(r / n_{k}\right)=f_{n_{k}}\left(r / n_{k}\right)$ for $0 \leqq r \leqq n_{k}$ and $f=0$ at other rationals. Then 


$$
\mathrm{V} B_{n_{k}} f=\mathrm{V} B_{n_{k}} f_{n_{k}} \geqq \frac{1}{10} A_{n_{k}} \rightarrow \infty \quad(n \rightarrow \infty) .
$$

Also for each $n$, if $n_{k}$ denotes the largest $n_{i}$ with $n_{i} \leqq n$,

$$
\begin{aligned}
\sum_{r=0}^{n} f\left(\frac{r}{n}\right) & \leqq \sum_{i=1}^{k} \sum_{r=0}^{n_{i}} f\left(\frac{r}{n_{i}}\right) \leqq \sum_{i=1}^{k} n_{i}^{1 / 2} A_{n_{i}} \\
& \leqq 2 n_{k}^{1 / 2} A_{n_{k}} \leqq 2 n^{1 / 2} A_{n} .
\end{aligned}
$$

Thus $f$ is bounded, V $B_{n} f$ unbounded and the sums are $O\left(n^{1 / 2} A_{n}\right)$. Part (c) follows from the same argument except that we let $f_{n} \equiv n^{-1 / 2} g_{n}$. If we remove the boundedness restriction on $f$ we can simply define $f$ to be $A_{n_{k}} n_{\boldsymbol{k}}^{1 / 2}$ at only one $r$ for each $n_{k}$ as long as $r / n_{k} \in I_{A}$, and 0 elsewhere.

4. An example. Define a skeleton by $f(p / q)=1 / q$ when $p$ and $q$ are relatively prime, and $p / q \in I_{A}$ for some $0<A<\frac{1}{2}$. Put $f=0$ on $[0,1]$ $-I_{A}$. By considering $n$ of the form $2^{N}$, it is easy to show $\sum_{r=0}^{n-1}|f((r+1) / n)-f(r / n)|$ is unbounded. Thus $f$ is not of bounded variation even with respect to the rationals, so that neither (1.2) nor Theorem (1.1) applies. However, Theorem (3.1.a) shows that V $B_{n} f \rightarrow 0$. To see this, let $n=q_{1}^{\alpha_{1}} \cdots q_{s}^{\alpha_{s}}$ ( $q_{i}$ prime). Now $f(r / n)$ can only assume the values $A_{1}^{-1} \cdots A_{d(n)-1}^{-1}$ where the $A_{i}>1$ are the divisors of $n$, and $d(n)$ is the number of such divisors. Each value $A_{i}^{-1}$ is assumed at most $A_{i}-1$ times so that

$$
\sum_{r=0}^{n}\left|f\left(\frac{r}{n}\right)\right|<\sum_{d(n)-1} 1<d(n) .
$$

But $d(n)=o\left(n^{\alpha}\right)$ for any $\alpha>0,\left[1\right.$, p. 260] and thus is $o\left(n^{1 / 2}\right)$.

\section{BIBLIOGRAPHY}

1. G. H. Hardy and E. M. Wright, An introduction to the theory of numbers, 3rd. ed., Clarendon Press, Oxford, 1954.

2. F. Herzog and J. D. Hill, The Bernstein polynomials for discontinuous functions, Amer. J. Math. 68 (1946), 109-124.

3. G. G. Lorentz, Bernstein polynomials, Univ. of Toronto Press, Ontario, 1953.

Wayne State University 